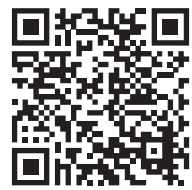

Enero - Marzo 2022 Vol. 2, núm. 1 / pp. 30-38

\section{Manejo del adenoma pleomorfo parotídeo recurrente provocado por anacrónica tumorectomía. Reporte de caso clínico y revisión analítica de literatura}

\author{
Management of recurrent parotid pleomorphous \\ adenoma caused by anachronistic lumpectomy. Clinical \\ case report and analytical literature review
}

Ludwing Manuel Alfredo Méndez Fuentes*
Palabras clave: Adenoma pleomorfo,

parotidectomía superficial,

lumpectomía, recurrencia, glándula parótida.

Keywords: Pleomorphic adenoma, superficial parotidectomy, lumpectomy, recurrence, parotid gland.
* Cirujano Bucal y Maxilofacial, Hospital Nacional «San Juan de

Dios») de Santa Ana. Presidente de la Asociación Salvadoreña de Cirugía Bucal y Maxilofacial. El Salvador.

Recibido: 25/09/2021 Aceptado: 12/10/2021

doi: 10.35366/103407

\section{RESUMEN}

El adenoma pleomorfo está relacionado con una alta tasa de recurrencia. En el pasado estuvo ligada a la tumorectomía. El propósito de este artículo es mostrar por qué fracasan las tumorectomías parotídeas mostrando sus consecuencias: el adenoma pleomorfo recurrente, difícil manejo por su multinodularidad y fibrosis, y posibilidad de malignización después de repetidas cirugías. El fracaso de este caso clínico fue por la tumorectomía que ocasionó dos recurrencia tumorales, ambas al año de operadas, con diseminación multinodular periférica. La parotidectomía total con preservación del nervio facial ofreció resultados favorables, tras 42 meses de evolución no hay parálisis facial y no existe evidencia de recurrencia tumoral. El tratamiento del adenoma pleomorfo recurrente no está estandarizado, pudiendo manejarse con parotidectomía total, seguimiento prolongado y uso de radioterapia adyuvante. Se recomienda no biopsias incisionales y parotidectomía convencional con conservación del nervio facia para evitar en la medida de lo posible recurrencia tumoral.

\section{ABSTRACT}

Pleomorphic adenoma is related to a high recurrence rate. In the past it was linked to lumpectomy. The purpose of this article is to show why lumpectomies fail by showing its consequences: recurrent pleomorphic adenoma, difficult management due to its multinodularity and fibrosis, possibility of malignancy after repeated surgeries. The failure of this case was due to the lumpectomy that caused two tumor recurrences, both a year after surgery, with peripheral multinodular dissemination. The total parotidectomy with sparing facial nerve, offered favorable results and with 42 months of evolution there is no facial palsy and there is no evidence of tumor recurrence. The treatment of recurrent pleomorphic adenoma is not standardized, and it can be managed with total parotidectomy, prolonged follow-up, and the use of adjuvant radiotherapy. It is recommended, not incisional biopsies, conventional parotidectomy with preservation of the facial nerve to avoid tumor recurrence as far as possible.

\section{INTRODUCCIÓN}

El adenoma pleomórfico es el tumor benigno que se encuentra con mayor frecuencia en la glándula parótida ${ }^{1}$ en $70-80 \%{ }^{2}$ Se compone de tejido epitelial, con patrones trabeculados, tubulares, sólidos, quístico, papilar compuesto por células mioepiteliales y un estroma derivado de células mioepiteliales modificadas, que pue- de ser: mixoide, mucoide, hialino, condroide y osteoide. ${ }^{3}$ Además, posee rasgos histológicos que lo hacen único, dichos rasgos son una cápsula incompleta, pequeñas excrecencias (pseudopodia), que se extienden a los tejidos adyacentes; estas características están relacionadas con su conducta clínica y su alta tasa de recurrencia con el pasar del tiempo. ${ }^{4}$ El tratamiento del adenoma pleomorfo ha evolucionado con el paso de los 
años. Entre los primeros cirujanos de glándula parótida están el alemán Johan Heyfelder, ${ }^{5}$ en 1847, quien preservó el nervio facial durante una parotidectomía. Erichsen, ${ }^{6}$ en 1869, evitó parálisis facial disecando el tumor a través de incisiones paralelas al tronco principal del VII par. Al cirujano rumano Codreanu $^{7}$ se le atribuye la primera parotidectomía total con preservación del nervio facial. Senn ${ }^{8}$ describió por primera vez la técnica conservadora de enucleación de los tumores parotídeos (enucleación intracapsular, tumorectomía), para evitar daño al nervio facial. Se afirma que al inicio del siglo XX y a finales de la década de 1930, el tratamiento del adenoma pleomórfico fue sustancialmente frustrante, debido a la tasa de recurrencia entre $20-45 \%$ y a la parálisis facial permanente, causada por la enucleación (tumorectomía); ;,9 incluso había recurrencia de hasta $50 \% .{ }^{10}$ Janes $^{11}$ y Bailey ${ }^{12}$ propusieron la identificación del tronco principal del nervio facial; como primer paso, antes de la resección de la glándula parótida. Hayes Martin ${ }^{13}$ concluyó que la mejor manera de evitar un daño permanente al nervio facial era identificando el tronco principal del nervio facial y, luego, realizando la parotidectomía superficial junto a la masa tumoral. Así, desde los años 50, la parotidectomía superficial es el estándar de referencia mundial en el manejo de los tumores del lóbulo superficial de la glándula parótida, ${ }^{14}$ la tasa de recurrencia disminuyó drásticamente, incluso hasta cero en algunos casos. ${ }^{2,15}$ La tumorectomía representa alta recurrencia, a menudo multinodular, del adenoma pleomorfo y su manejo quirúrgico es un verdadero reto, ya que está asociada con daño del nervio facial. ${ }^{16}$ Un apropiado abordaje para el adenoma pleomorfo es imprescindible, no sólo por su potencial de recurrencia,

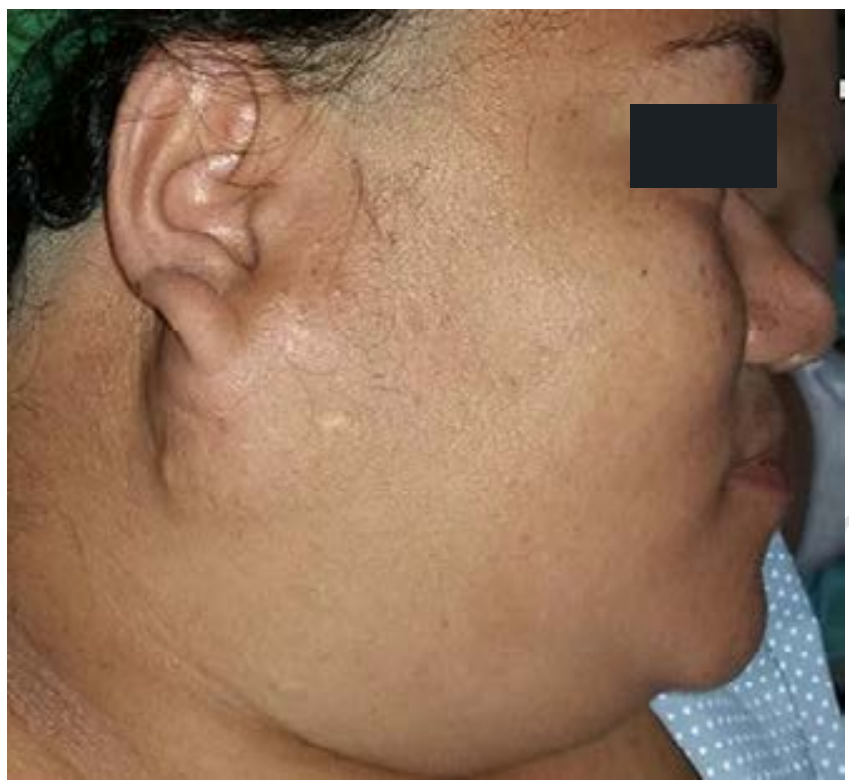

Figura 1: Imagen prequirúrgica. sino también porque va adquiriendo características de malignización lentamente, después de repetidas recurrencias. ${ }^{17}$ Así que los adenomas pleomorfos, en la práctica quirúrgica contemporánea, ya no son enucleados, y la parotidectomía superficial o total, junto a la disección extracapsular son los tratamientos de elección ante el aparecimiento de neoplasias parotídeas ${ }^{16}$ y sería la forma más segura de prevenir en un porcentaje aceptable la recurrencia neoplásica.

El propósito de este artículo es mostrar por qué fracasan las tumorectomías parotídeas mostrando sus consecuencias nefastas: el adenoma pleomorfo recurrente, su difícil manejo por multinodularidad y fibrosis, y la posibilidad de malignización después de repetidas cirugías; además, se analizan algunas alternativas del manejo de recurrencias tumorales a nivel mundial. Las tumorectomías continúan realizándose en El Salvador, y se demuestra en el caso clínico de una paciente sometida en dos ocasiones a enucleación intracapsular (tumorectomía) parotídea por otra especialidad quirúrgica, demostrando que la parotidectomía total con conservación del nervio facial, continúa siendo el tratamiento quirúrgico efectivo en tumores primarios de la glándula parótida y en el manejo de recurrencias tumorales.

\section{Material y métodos}

El presente estudio es un reporte de caso clínico más revisión analítica de literatura, en donde se evalúa el caso de una paciente de 24 años de edad sin antecedentes patológicos, quien se presenta en el año 2008 a un hospital nacional en El Salvador, presentando un nódulo parotídeo en lado derecho

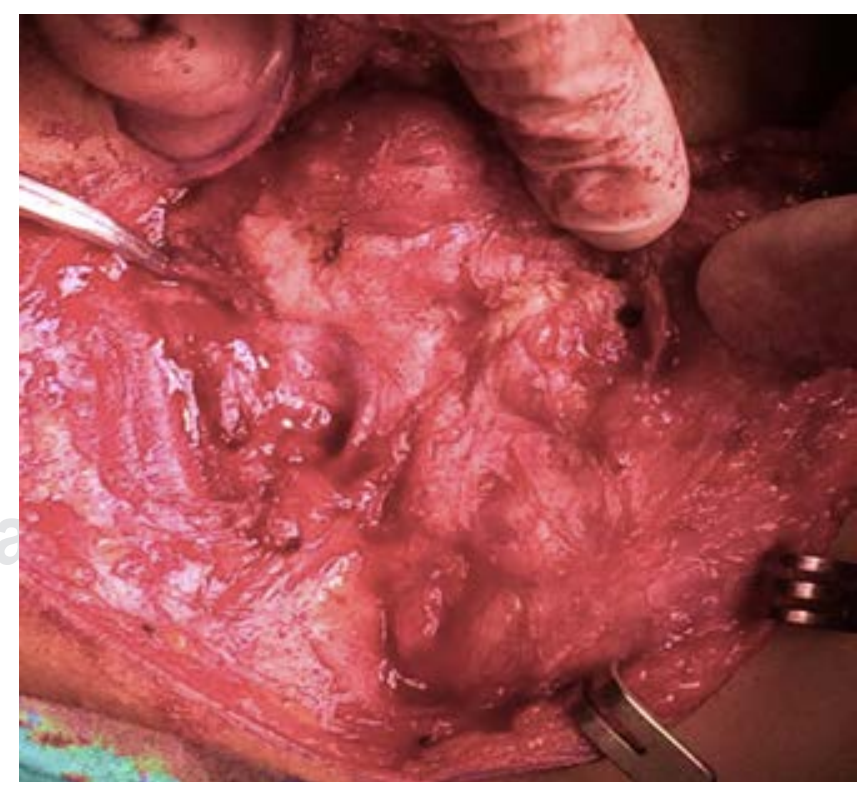

Figura 2: Abordaje y localización de la lesión. 


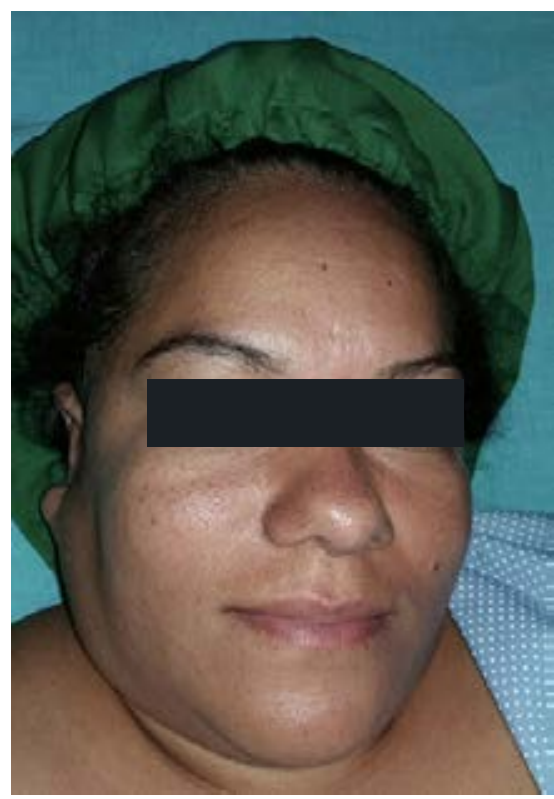

Figura 3:

Foto frontal preoperatoria.

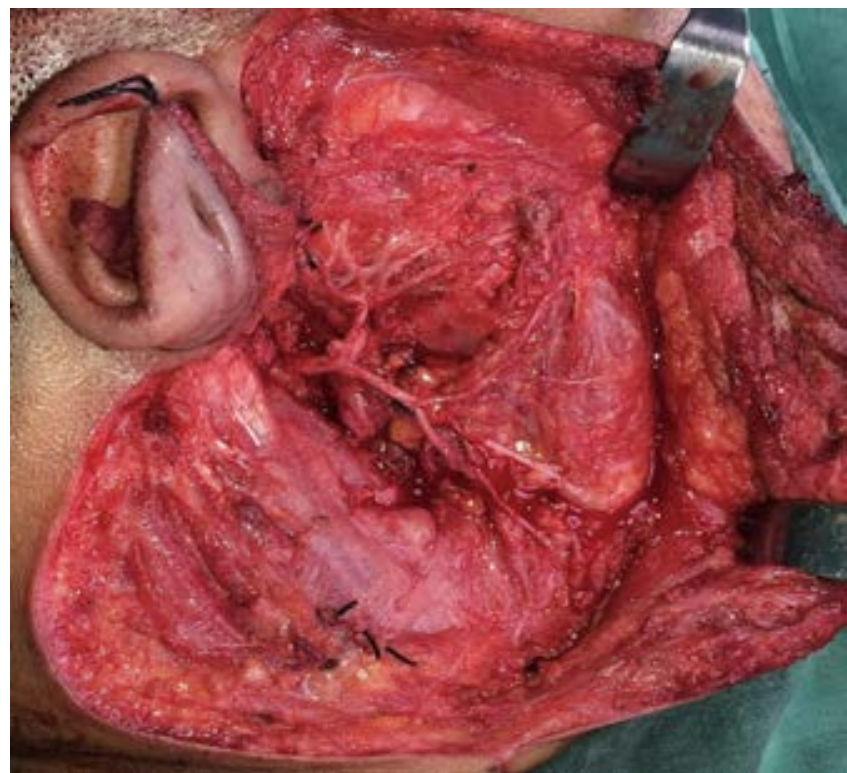

Figura 4: Desección y remoción de la lesión cuidando reparos anatómicos.

de crecimiento lento, no refería dolor, como tampoco presentaba parálisis facial. Fue evaluada por otra especialidad quirúrgica y fue sometida a tumorectomía parotídea bajo anestesia general, se manda pieza quirúrgica a Servicio de Anatomía Patológica, dando como resultado adenoma pleomorfo. En el año 2009 notó un nuevo crecimiento indoloro en sitio de la operación, pero no acudió a tratamiento. Fue hasta 2013 que consulta con la misma especialidad quirúrgica que la trató al

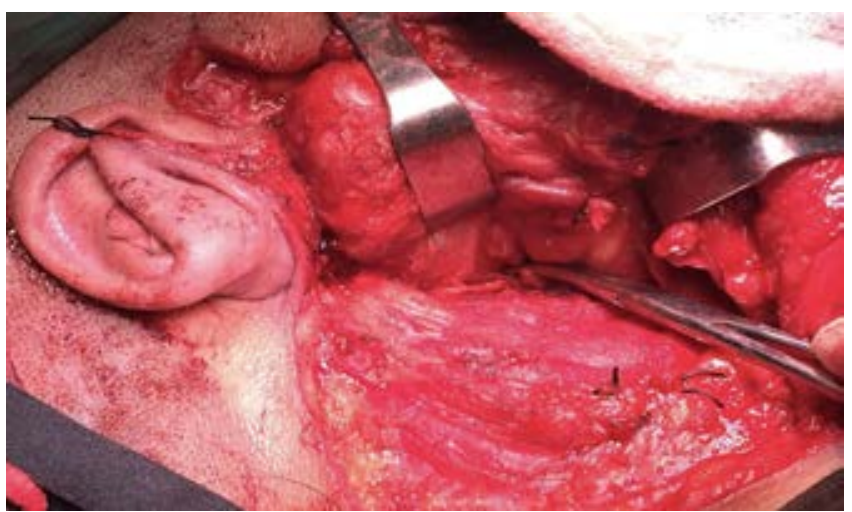

Figura 5: Eliminación por planos de la lesión.
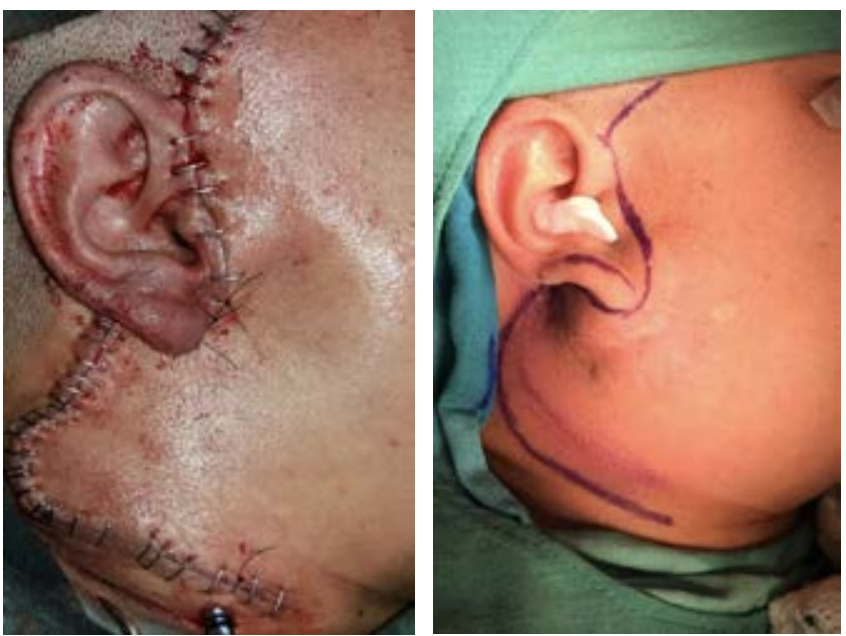

Figura 6: Sutura y cierre en el postoperatorio inmediato.

inicio y fue sometida a nueva tumorectomía parotídea bajo anestesia general y una nueva recidiva aparece en el 2014, siendo dejada en observación. En el 2017 fue evaluada de nuevo, siendo evidente el crecimiento tumoral, al cual se le realizó punción aspirativa con aguja fina (PAAF), arrojando como resultado adenoma pleomorfo recurrente (residual). En 2018, la paciente fue evaluada por primera vez en la práctica privada de Cirugía Bucal y Maxilofacial en Santa Ana, El Salvador. Se evaluó masa multinodular, no dolorosa, no estaba fijada a los tejidos profundos ni a la piel, existía adecuada movilidad, había coloración normal de la piel sin ulceración, sólo existía cicatriz hipertrófica. No se observó parálisis facial ni movimientos espasmódicos de la musculatura facial. Se evalúa ultrasonografía observando neoplasia del tamaño de $4.6 \times 2.2 \mathrm{~cm}$, razón por la cual se programa parotidectomía total bajo anestesia general. Se realizó abordaje de Blair modificado con levantamiento de colgajo de SMAS (sistema 
músculo-aponeurótico superficial), identificación de tronco principal del nervio facial con resección de lóbulo superficial acompañando toda la masa multinodular y resección de lóbulo profundo, además se resecó piel con cicatriz hipertrófica, por la posibilidad de existir alguna siembra tumoral cutánea. Se coloca dreno Jackson-Pratt, cierre de SMAS con vicryl 3-0 y cierre de piel con grapas y nylon 4-0.

\section{Resultados}

La cirugía fue realizada sin complicaciones, fue removida toda la glándula parótida junto a toda la masa multinodular, preser-

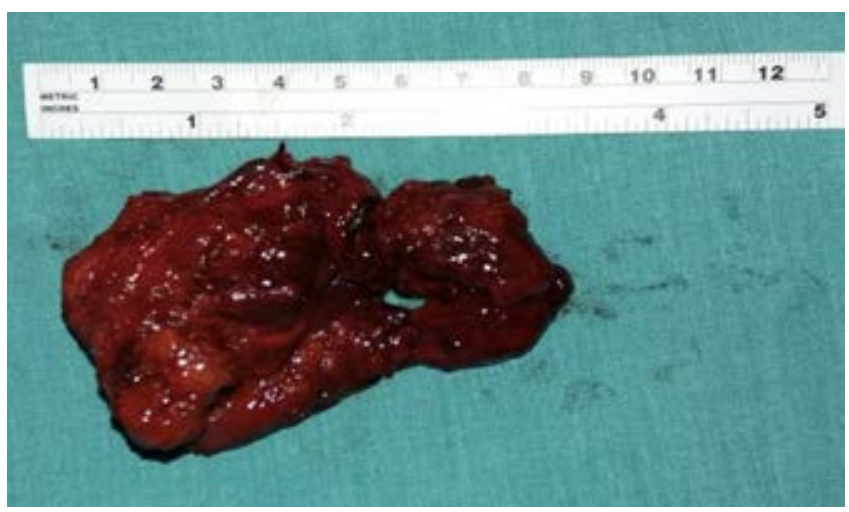

Figura 7: Lesión extraída.

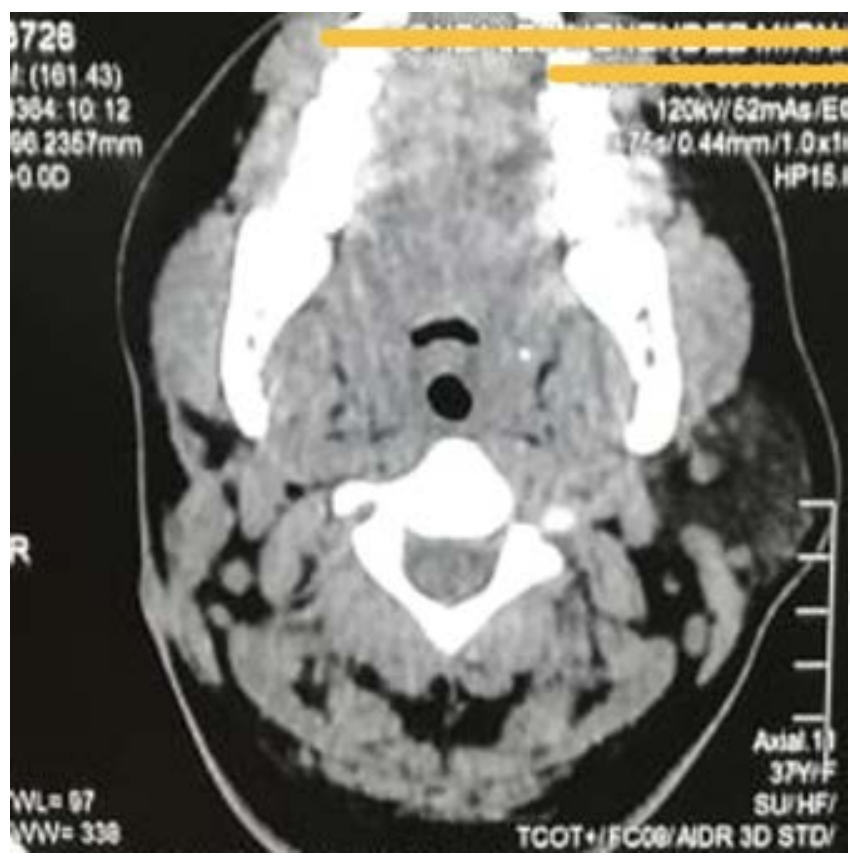

Figura 8: Tomografía preoperatoria. vando la integridad del nervio facial, a pesar de no contar con estimulador eléctrico. Durante la primera semana se evaluó a diario, el dreno permaneció 10 días y se retiraron grapas e hilos de nylon. No hubo infección ni dehiscencia de la herida (Figuras 1 a 12). Los primeros 15 días se observó limitación en los movimientos faciales, los cuales se fueron recuperando al finalizar el primer mes, recuperando por completo su mímica facial. Los primeros seis meses se evaluaba mensualmente, luego del primer año, cada seis meses. Hubo adormecimiento de la región preauricular por sección del nervio auricular mayor. A la fecha, son tres años y seis meses de evolución, se evalúa clínica y tomográficamente, no hay parálisis facial, no hay evidencia de recurrencia tumoral, tampoco existe evidencia clínica de síndrome de Frey. Al momento sólo hay cicatriz hipertrófica.

\section{Discusión}

Una de las complicaciones más temidas del adenoma pleomorfo es la posible recurrencia tumoral, ${ }^{2}$ que puede aparecer después de la primera cirugía, en el intervalo de 7-10 años ${ }^{18}$ y en un periodo de tiempo más corto después de la tumorectomía. ${ }^{19}$ Antes de la década de 1940, las recidivas oscilaban entre $20-45 \%$ y $50 \% .^{10,18-20}$ Esto se debía a que el tratamiento del adenoma pleomorfo parotídeo era la enucleación intracapsular o tumorectomía. ${ }^{9,19}$ A partir de los hallazgos de Patey, ${ }^{21}$ se estimó que una causa de recurrencia eran los pseudópodos o pequeños lóbulos, encontrados por fuera de la presunta cápsula tumoral (pseudocápsula), que durante una enuclea-

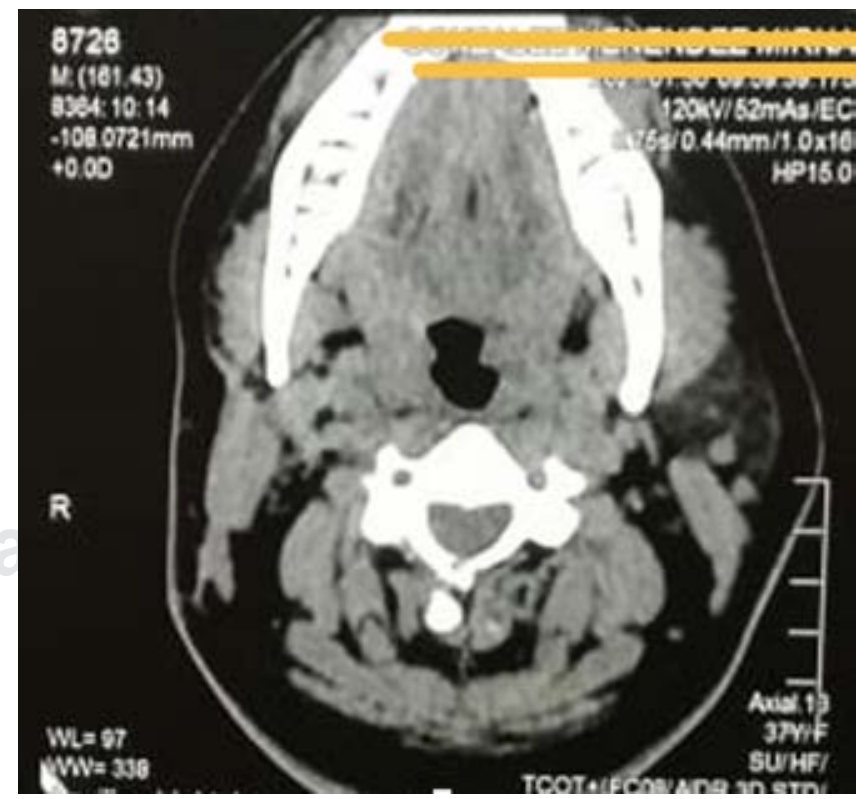

Figura 9: Tomografía postoperatoria. 
Edad: 34 años

Médico: Dr. Ludwing Méndez

Fecha de recepción: 12 de febrero de 2018

Fecha de reporte: 15 de febrero de 2018

Estudio de: tumor de glándula parótida derecha. Estudio macroscópico: muestra de $9.0 \times 5.5 \mathrm{~cm}$. A los cortes de aspecto multinodular, blanquecina y renitente. Estudio microscópico: glándula salival con fibrosis, moderada inflamación crónica multifocal y nódulos de varios tamaños de neoplasia mixta benigna constituida por estroma mixoide y mucocondroide y nidos de células mioepiteliales y epiteliales. Hay también estructuras nerviosas y músculo-esquelético firmemente adheridos por fibrosis. No se encontró malignidad en lo examinado. Límites quirúrgicos sanos.

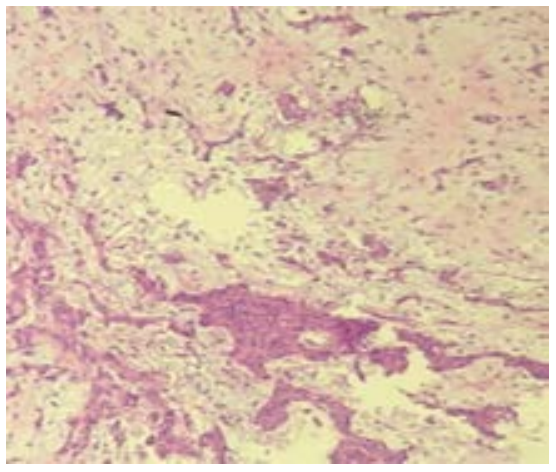

Diagnóstico: *Adenoma pleomórfico recurrente, glándula parótida derecha (extirpación). *Fibrosis e inflamación crónica, glándula parótida derecha.
BA-18-107 Médico: Dr. Ludwing Méndez

(1) Fecha de recepción: 12 de febrero de 2018

Fecha de reporte: 15 de febrero de 2018

Estudio de: fragmentos de piel de región parotídea derecha. Estudio macroscópico:

4 fragmentos de piel de:

1) $2.3 \times 0.5 \mathrm{~cm}$, extirpado a $0.5 \mathrm{~cm}$ de profundidad.

2) $2.5 \times 0.5 \mathrm{~cm}$ extirpado a $0.5 \mathrm{~cm}$ de profundidad.

3) $7.0 \times 0.6 \mathrm{~cm}$, extirpado a $0.7 \mathrm{~cm}$ de profundidad.

4) $7.0 \times 3.0 \mathrm{~cm}$, extirpado a $0.8 \mathrm{~cm}$ de profundidad.

\section{Estudio microscópico:}

En los 4 fragmentos se observa:

Epidermis: leve atrofia y escasas erosiones.

Dermis y tejido celular subcutáneo: difusa fibrosis y moderada inflamación aguda y crónica multifocal.

No se encontró malignidad en lo examinado.

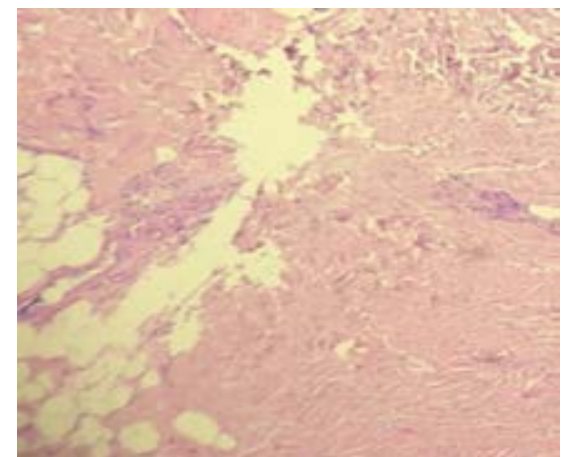

Diagnóstico: *fibrosis e inflamación aguda y crónica, piel de región parotídea derecha (4 fragmentos).
(2)

\section{Figura 10:}

Estudio histopatológico. ción descontrolada pueden desprenderse o dislocarse y ser dejados en el tejido parotídeo normal (recurrencia tumoral uninodular) ${ }^{18}$ junto a los nódulos satélites. ${ }^{2,17}$ La ruptura intraoperatoria de la cápsula tumoral con su respectivo derrame de tejido neoplásico en los tejidos adyacentes también es otra causa de recurrencia tumoral (recurrencia tumoral multinodular), ${ }^{19,22,23}$ similar como sucede con las biopsias incisionales, razón por la cual están indefectiblemente contraindicadas. ${ }^{9,24}$ A partir de los resultados de Janes, ${ }^{11}$ Bailey ${ }^{12}$ y Hayes Martin, ${ }^{13}$ ya en 1950, y debido al fracaso de la tumorectomía, la parotidectomía superficial o total con preservación del nervio facial se establece como la norma de referencia mundial para el manejo de las neoplasias parotídeas benignas y malignas de bajo grado. 2,9,25

Se observó que la parotidectomía superficial reducía dramáticamente las altas tasas de recurrencia que ocurrían con la enucleación simple del adenoma pleomorfo; ${ }^{26}$ sin embargo, no se eliminan del todo las posibilidades de recurrencia. Se establece en general que en la actualidad hay una tasa de recurrencia hasta debajo de $4 \%{ }^{22,27-29}$ Las recurrencias tumorales tienen más tendencia a ser multino- dulares que un nódulo solitario. ${ }^{30}$ Algo que podría explicar la alta incidencia de una segunda recurrencia tumoral es que microscópicamente pueden llegar a existir hasta 100 nódulos más pequeños que 1 milímetro, ${ }^{18}$ predominando en el subtipo mixoide hasta en $80 \%{ }^{3}$ Dicho subtipo se caracteriza por tener una cápsula incompleta y nódulos satélites, los cuales pueden escapar durante la enucleación y ser dejados en los tejidos. ${ }^{27,29}$ Muchos estudios señalan que a los pacientes jóvenes se les realizan tratamientos más conservadores, siendo ésta la causa de las recurrencias tumorales en este grupo etario. ${ }^{31}$ Por eso se justifica la parotidectomía convencional para los adenomas pleomorfos, ya que se observan tasas de recurrencia de cero, como la reportada por Donovan. ${ }^{9}$ Rodriguez-Bigas ${ }^{15}$ reportó $0.7 \%$ de recidiva que fue un caso de tumorectomía. Maynard ${ }^{32}$ en una serie de 336 parotidectomías, con seguimiento hasta de 20 años, sólo reporta una recurrencia. Woods ${ }^{33}$ reportó 86 parotidectomías con seguimiento entre cinco y 13 años y no hubo reporte de recurrencias tumorales ni de parálisis faciales permanentes. O'Dwyer ${ }^{34}$ reportó 274 parotidectomías en donde hubo nueve recurrencias (3.2\%), en el intervalo de 
5-20 años, además realizó 34 parotidectomías para tumores recurrentes, de las cuales hubo 10 recurrencias (29\%) en el periodo de cinco años, concluyendo que la recurrencia no es común, si se realiza parotidectomía superficial o total para un tumor primario. Stevens ${ }^{35}$ reportó 100 parotidectomías, tratadas con parotidectomía superficial, de las cuales 72 eran tumores primarios, obteniendo una tasa de cero recurrencias y ningún daño al VII par; los otros 28 pacientes con recurrencia tumoral por tumorectomía y con seguimiento entre 1-15 años; se reportaron cinco recurrencias y cuatro transformaciones malignas. Leverstein ${ }^{2}$ reportó una tasa de recurrencia de $0.8 \%$ de 246 pacientes realizando parotidectomías convencionales y conservadoras, pero ninguna tumo-

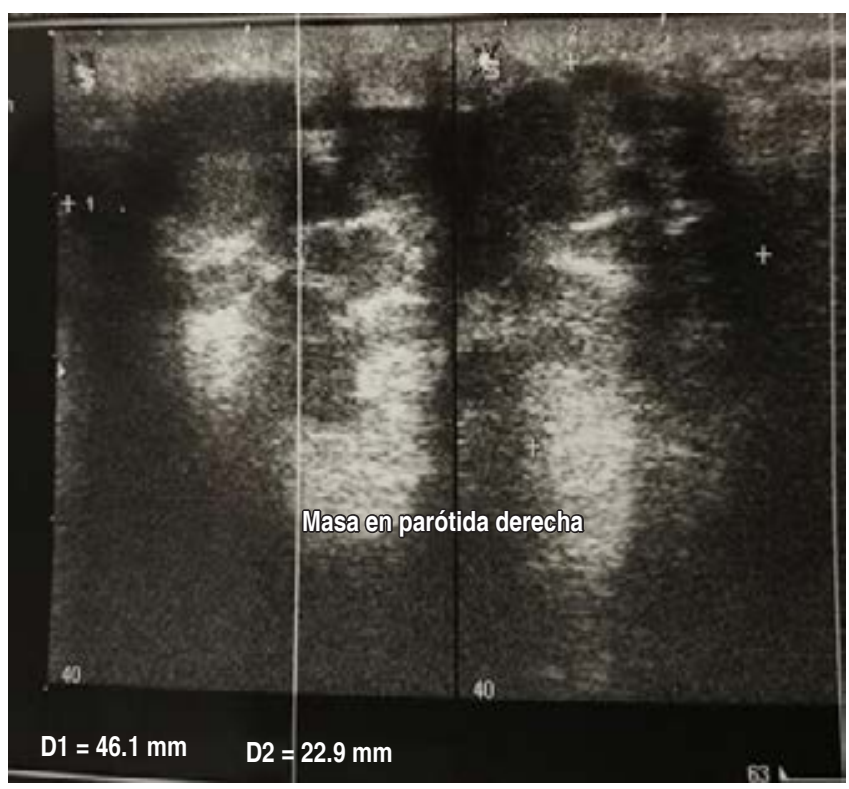

Figura 11: Imagenología de masa en parótida derecha. rectomía. Papadogeorgakis, ${ }^{36}$ después de la parotidectomía superficial reporta una tasa de recidiva de $3 \%$.

A causa del riesgo de recurrencia tumoral, un seguimiento por muchos años deberá ser llevado a cabo, ya que hay reportes recientes de recurrencia tumoral después de 15 años. ${ }^{37} \mathrm{Sin}$ embargo, un reporte de State y Grage ${ }^{38}$ afirma que hasta después de 20 años de estar libre de tumor apareció recurrencia. Se aclara que tanto en las técnicas formales de parotidectomía (parotidectomía superficial y total), como en las conservadoras (parotidectomía parcial superficial y disección extracapsular) existen tasas variables de recurrencia tumoral. ${ }^{22} \mathrm{Y}$ esto es debido a que la cápsula del tumor, sin importar la técnica quirúrgica, se expone hasta en $60 \%$ de los casos, cuando una o más ramas del nervio facial está en contacto directo con la cápsula del adenoma pleomorfo, requiriendo enucleación, siendo así la resección en bloque imposible y pudiendo romper la cápsula con respectiva siembra tumoral, explicando así la recurrencia después de una parotidectomía superficial. ${ }^{18,36}$ Después de una recurrencia neoplásica, se incrementaría la posibilidad de malignización, ${ }^{10}$ que ha sido reportada hasta en $3.3 \% .{ }^{17,39}$ Una de las mutaciones del adenoma pleomorfo es su transformación en carcinoma ex-adenoma pleomorfo, ${ }^{40}$ hasta en 1.8-6.2\%. ${ }^{28}$ Dicha malignización no sólo se observa en tumores benignos recurrentes, sino también en neoplasias que no han sido tratadas, dicho porcentaje puede ser de $10 \%$ al transcurrir 15 años y de $20 \%$ pasados los 30 años. ${ }^{41}$ Otra adversidad de la cirugía de un adenoma pleomorfo recurrente es que exista un alto riesgo de daño al nervio facial, a causa de la fibrosis alrededor del nervio, ${ }^{19}$ siendo la parálisis permanente del nervio facial el principal riesgo al tratar adenomas pleomórficos recurrentes. ${ }^{31} \mathrm{~A}$ causa de la complejidad del problema se podrían definir tres abordajes para las recurrencias tumorales: ${ }^{31}$

1. Escisión radical con sacrificio del nervio facial, con su alta morbilidad postoperatoria.
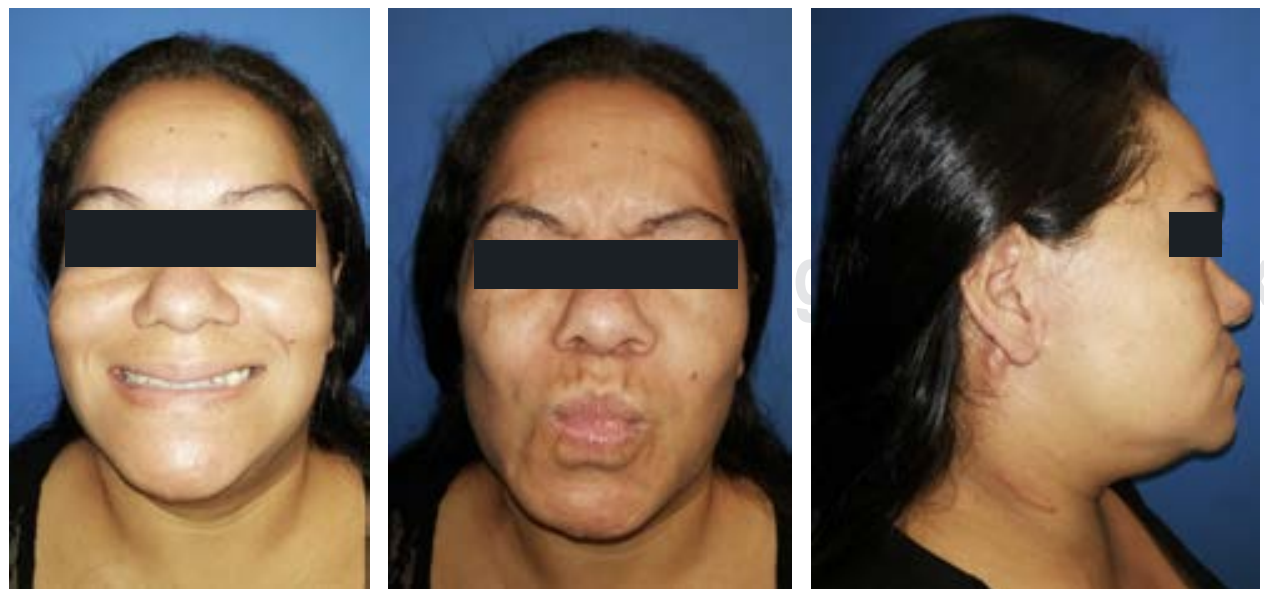

Figura 12:

Secuencia de fotografías postoperatorias demostrando función adecuada. 
2. Resección con disección y preservación del nervio facial, junto a la escisión cicatricial de la primera cirugía. ${ }^{18}$ Aunque la preservación del nervio facial, en este contexto, se podría relacionar a altas tasas de recurrencia.

3. La radioterapia adyuvante, recomendada por muchos autores como una modalidad de rutina parece erradicar residuos microscópicos.

Hay evidencia de que la radioterapia en pacientes que no son aptos para cirugía y la administrada en el postquirúrgico, ante una incompleta remoción del adenoma parotídeo y en la recurrencia multinodular, puede mejorar el control de la enfermedad. ${ }^{3,18,42}$ Samson $^{43}$ reportó $94 \%$ libre de recidiva tumoral con preservación del nervio facial y radioterapia adyuvante con un seguimiento de 5.9 años. En otras series retrospectivas se reporta un mejor control local con cirugía y radioterapia que sólo la cirugía, hasta en 94\% después de 20 años. ${ }^{18,44}$ En ausencia de radioterapia postquirúrgica, la recurrencia tumoral fue de $32.5 \%$ después de la primera cirugía, $7.1 \%$ después de la segunda cirugía y $1.6 \%$ después de la tercera. ${ }^{45}$ Pero también existe alguna renuencia respecto a la radioterapia, ya que puede inducir a un segundo tumor maligno en el tejido glandular remanente o en otros órganos de cabeza y cuello. ${ }^{17}$ También transformación maligna dentro del adenoma pleomorfo recurrente, producto de un posible efecto estimulador de la radioterapia. ${ }^{3}$ Por lo tanto, un seguimiento a largo plazo es necesario, independientemente del abordaje realizado, ya que la posibilidad de una segunda recurrencia después de uno, dos, cinco, 10 y 15 años fue estimado 16, 23, 42, 60 y $75 \%$, respectivamente. ${ }^{46}$

\section{Conclusiones}

El manejo de la recurrencia del adenoma pleomorfo parotídeo no tiene un protocolo concluyente, sin embargo, hay recomendaciones precisas como:

1. La observación de la recurrencia neoplásica en pacientes ancianos o con enfermedades crónicas y en casos seleccionados esperar a que lesiones pequeñas crezcan: «esperar y ver».

2. Evaluar con resonancia magnética, ya que es la imagen de elección en las recurrencias tumorales del adenoma pleomorfo. ${ }^{18}$

3. Inexorablemente, preservación de la integridad del nervio facial, ya que haciéndolo produciría una tasa de recurrencia baja de 15\%, la radioterapia sería útil en estos casos, sobre todo en recurrencia multinodular.

4. El riesgo de daño nervioso permanente es de $15 \%$ en la primera reoperación y $30 \%$ en la segunda, por tanto, es imprescindible el uso de estimulador eléctrico, ya que podría evitar un daño del nervio facial permanente.
5. La tasa de malignización en la práctica es baja y su desarrollo es principalmente por múltiples intervenciones, ya sean quirúrgicas o radioterápicas. También, Witt, ${ }^{18}$ Sam$\operatorname{son}^{43}$ y Kerawala ${ }^{3}$ abogan por radioterapia adyuvante, sin olvidar la posibilidad de estigmas cutáneos, xerostomía, osteoradionecrosis, otitis y riesgo de aparecimiento de un segundo tumor como carcinoma de células escamosas y fibrosarcomas.

6. Stennert y su equipo ${ }^{47}$ recomiendan que sin importar la recurrencia, ya sea nodular o multinodular después de una parotidectomía superficial, sea tratada con parotidectomía total, ya que puede reducir pero no prevenir en su totalidad los residuos microscópicos dejados en la cirugía.

7. Piorkowsky ${ }^{48}$ menciona el sacrificio del nervio facial cuando hay múltiples recurrencias o cuando ha fallado la radioterapia, incluso así no se puede asegurar que no habrá recurrencia.

8. La recurrencia multinodular produce una recurrencia subsecuente de $45 \%$ sólo cirugía y hasta $4 \%$ cirugía y radioterapia. La recurrencia uninodular con radioterapia adyuvante con cirugía o sin ella, produce una tasa de recurrencia de $15 \%$.

9. Se debe asegurar, en la medida de lo posible, el éxito en la primera cirugía del adenoma pleomorfo, como mencionó O'Dwyer: ${ }^{34}$ la recurrencia no es común, si se realiza parotidectomía superficial o total para un tumor primario.

La tumorectomía parotídea continúa siendo realizada en la actualidad en El Salvador, a pesar que desde la década de 1950 la parotidectomía superficial y/o total con preservación del nervio facial quedó establecida como el estándar de oro para el manejo de neoplasias benignas y malignas de bajo grado de la glándula parótida. ${ }^{9,15} \mathrm{El}$ fracaso de este caso clínico fue por la tumorectomía practicada en dos ocasiones, ya que hubo recurrencia tumoral con diseminación multinodular en la periferia, en las dos ocasiones la recurrencia apareció al año de operada. A la fecha, la parotidectomía total con preservación del nervio facial, ha ofrecido resultados favorables, no vistos en los tratamientos anteriores y con 42 meses de evolución no hay parálisis facial, no hay síndrome de Frey, no existe evidencia clínica ni tomográfica de recurrencia tumoral, aunque se deberá dar seguimiento por muchos años. Por estas razones adversas Rodriguez-Bigas, ${ }^{15}$ refiere que la tumorectomía debe ser condenada por sus resultados ignominiosos y nefastos. La relativa baja incidencia del adenoma pleomorfo recurrente menos del $4 \%$, el seguimiento clínico por muchos años y el no concluyente uso de radioterapia adyuvante, hace que el conocimiento profundo de todos los aspectos de esta enfermedad sea un alto desafío. 


\section{ReferenCias}

1. Zernial O, Springer IN, Warnke P, Harle F, Risick C, Wiltfang J. Longterm recurrence rate of pleomorphic adenoma and postoperative facial nerve paresis (in parotid surgery). J Craniomaxillofac Surg. 2007; 35: 189-192.

2. Leverstein H, van der Wal JE, Tiwari RM, van der Waal I, Snow GB. Surgical management of 246 previously untreated pleomorphic adenomas of the parotid gland. Br J Surg. 1997; 84: 399-403.

3. Kerawala C, Brennan PA, Cascarini L, Godden D, Coombes D, McCaul J. Management of tumour spillage during parotid surgery for pleomorphic adenoma. Br J Oral Maxillofac Surg. 2014; 52: 3-6.

4. Foresta E, Torroni A, Di Nardo F, et al. Pleomorphic adenoma and benign parotid tumors: extracapsular dissection vs superficial parotidectomy review of literature and meta-analysis. Oral Surg Oral Med Oral Pathol Oral Radiol. 2014; 117: 663-676.

5. Heyfelder JF. Die versuche mit dem schwefeläther. Erlangen: Verlag Von Carl Heyder; 1847.

6. Erichsen JE. The science and art of surgery. Philadelphia: Collins Printer; 1869.

7. Dell'Aversana Orabona G, Bonavolonta P, laconetta G, Forte R, Califano L. Surgical management of benign tumors of the parotid gland: extracapsular dissection versus superficial parotidectomy--our experience in 232 cases. J Oral Maxillofac Surg. 2013; 71: 410-413.

8. Senn N. The pathology and surgical treatment of tumors. Philadelphia, PA: PW. B. Saunders; 1895.

9. Donovan D, Conley J. Capsular significance in parotid tumor surgery: Reality and myths of lateral lobectomy. Laryngoscope. 1984; 94: 324-329.

10. Work WP. Therapy of salivary gland tumors. Arch Oto Head Neck Surg. 1966; 83: 89-91.

11. Janes RM. The treatment of tumours of the salivary glands by radical excision. Can Med Assoc J. 1940; 43: 554-559.

12. Bailey H. Treatment of tumours of the parotid gland with special reference to total parotidectomy. Br J Surg. 1941; 28: 337-346.

13. Martin $\mathrm{H}$. The operative removal tumors of the parotid salivary gland. Surgery. 1952; 31: 670-682.

14. Liu H, Pei J, He Y, Lan X, Sun R, Deng T, et al. Comparison of functional change in parotid gland after surgical excision of pleomorphic adenoma by two different types of parotidectomy. Oral Surg Oral Med Oral Pathol Oral Radiol. 2016; 1223 85-91.

15. Rodriguez-Bigas MA, Sako K, Razack MS, Shedd DP, Bakamjian VY. Benign parotid tumors: a 24 year experience. J Surg Oncol. 1991; 46: 159-161.

16. Kanatas A, Ho MWS, Mücke T. Current thinking about the management of recurrent pleomorphic adenoma of the parotid: a structured review. Br J Oral Maxillofac Surg. 2018; 56: 243-248.

17. Becelli R, Morello R, Renzi G, Matarazzo G, Dominici C. Recurrent pleomorphic adenoma of the parotid gland: role of neutron radiation therapy. J Craniofac Surg. 2012; 23: e449-e450.

18. Witt RL, Eisele DW, Morton RP, Nicolai P, Poorten VV, Zbaren P. Etiology and management of recurrent parotid pleomorphic adenoma. Laryngoscope. 2015; 125: 888-893.

19. Niparko JK, Beauchamp ML, Krause CJ, Baker SR, Work WP. Surgical treatment of recurrent pleomorphic adenoma of the parotid gland. Arch Otolaryngol Head Neck Surg. 1986; 112(11): 1180-1184.

20. Albergotti WG, Nguyen SA, Zenk J, Gillespie MB. Extracapsular dissection for benign parotid tumors: a meta-analysis. Laryngoscope. 2012; 122: 1954-1960.

21. Patey DH, Thackray AC. The treatment of parotid tumours in the light of a pathological study of parotidectomy material. Br J Surg. 1958; 45: 477-487.
22. Witt RL. The significance of the margin in parotid surgery for pleomorphic adenoma. Laryngoscope. 2002; 112: 2141-2154.

23. Park GC, Cho KJ, Kang J, Roh JL, Choi SH, Kim SY, et al. Relationship between histopathology of pleomorphic adenoma in the parotid gland and recurrence after superficial parotidectomy. J Surg Oncol. 2012; 106: 942-946.

24. Maahs GS, Oppermann Pde O, Maahs LG, Machado Filho G, Ronchi AD. Parotid gland tumors: a retrospective study of 154 patients. Braz J Otorhinolaryngol. 2015; 81: 301-306.

25. Beahrs $\mathrm{OH}$. Parotid tumors and their surgical management. Am Surg. 1957; 23 (10): 891-899.

26. Buxton R, Maxwell J, French A. Surgical treatment of epithelial tumors of the parotid gland. Surg Gynecol Obstet. 1953; 97: 401-416.

27. Zbaren P, Stauffer E. Pleomorphic adenoma of the parotid gland: histopathologic analysis of the capsular characteristics of 218 tumors. Head Neck. 2007; 29: 751-757.

28. Valstar MH, Ridder M, van den Broek EC, Stuiver MM, van Dijk BAC, van Velthuysen MLF, et al. Salivary gland pleomorphic adenoma in the Netherlands: A nationwide observational study of primary tumor incidence, malignant transformation, recurrence, and the risk factors for recurrence. Oral Oncol. 2017; 66: 93-99.

29. Zbaren P, Vander Poorten V, Witt RL, Woolgar JA, Shaha AR, Triantafyllou A, et al. Pleomorphic adenoma of the parotid: formal parotidectomy or limited surgery? Am J Surg. 2013; 205: 109-118.

30. Rowley H, Murphy M, Smyth D, et al. Recurrent pleomorphic adenoma: uninodular versus multinodular disease. Ir J Med Sci. 2000; 169: 201-203.

31. Renehan A. Treatment of recurrent pleomorphic adenomas. In: McGurk M, Combes J. Controversies in the management of salivary gland disease. 2nd ed. Oxford University Press; 2013.

32. Maynard JD. Management of pleomorphic adenoma of the parotid. Br J Surg. 1988; 75: 305-308.

33. Woods JE. Parotidectomy versus limited resection for benign parotid masses. Am J Surg. 1985; 149: 749-750.

34. O'Dwyer PJ, Farrar WB, Finkelmeier WR, McCabe DP, James AG. Facial nerve sacrifice and tumor recurrence in primary and recurrent benign parotid tumors. Am J Surg. 1986; 152: 442-445.

35. Stevens KL, Hobsley M. The treatment of pleomorphic adenomas by formal parotidectomy. Br J Surg. 1982; 69: 1-3.

36. Papadogeorgakis N. Partial superficial parotidectomy as the method of choice for treating pleomorphic adenomas of the parotid gland. Br J Oral Maxillofac Surg. 2011; 49: 447-450.

37. Abu-Ghanem Y, Mizrachi A, Popovtzer A, et al. Recurrent pleomorphic adenoma of the parotid gland: institutional experience and review of the literature. J Surg Oncol. 2016; 114: 714-718.

38. State D, Grage TB. Surgical treatment of parotid tumors. Curr Probl Surg. 1966; 3: 3-67.

39. Andreasen S, Therkildsen MH, Bjorndal K, et al. Pleomorphic adenoma of the parotid gland 1985-2010: a Danish nationwide study of incidence, recurrence rate, and malignant transformation. Head Neck. 2016; 38(suppl 1): E1364-E1369.

40. Makeieff M, Pelliccia P, Letois F, et al. Recurrent pleomorphic adenoma: results of surgical treatment. Ann Surg Oncol. 2010; 17: 3308-3313.

41. O'Regan B, Bharadwaj G. Tumour recurrence after surgical removal of parotid pleomorphic salivary adenoma using a retrograde facial nerve dissection technique. Br J Oral Maxillofac Surg. 2012; 50: 417-419.

42. Renehan A, Gleave EN, McGurk M. An analysis of the treatment of 114 patients with recurrent pleomorphic adenomas of the parotid gland. Am J Surg. 1996; 172: 710-714. 
43. Samson MJ, Metson R, Wang CC, et al. Preservation of the facial nerve in the management of recurrent pleomorphic adenoma. Laryngoscope. 1991; 101: 1060-1062.

44. Wallace AS, Morris CG, Kirwan JM, Werning JW, Mendenhall WM. Radiotherapy for pleomorphic adenoma. Am J Otolaryngol. 2013; 34: 36-40.

45. Phillips PP, Olsen KD. Recurrent pleomorphic adenoma of the parotid gland: report of 126 cases and a review of the literature. Ann Otol Rhinol Laryngol. 1995; 104: 100-104.

46. Wittekindt C, Streubel K, Arnold G, et al. Recurrent pleomorphic adenoma of the parotid gland: analysis of 108 consecutive patients. Head Neck. 2007; 29: 822-828.
47. Stennert E, Wittekindt C, Klussmann JP, Arnold G, Guntinas-Lichius O. Recurrent pleomorphic adenoma of the parotid gland: a prospective histopathological and immunohistochemical study. Laryngoscope. 2004; 114: 158-163.

48. Piorkowski RJ, Guillamondegui OM. Is aggressive surgical treatment indicated for recurrent benign mixed tumors of the parotid gland? Am J Surg. 1981; 142: 434-436.

Correspondencia:

Ludwing Manuel Alfredo Méndez Fuentes

E-mail: ludwingmendezzmaxilo@gmail.com 